\title{
Soluble curcumin in the prevention of diabetic retinopathy via modulation of anti-oxidant activity and genetic pathways - in vivo model
}

\begin{abstract}
The aim of the present study was to investigate the protective effects of Curcuminoids from regular curcumin Powder (95\% Curcuminoids), and UltraSOL TM Nutrient System with curcumin (CurcuWINTM Turmeric Extract, 20\% curcuminoids) in dry powder forms on retina and metabolic markers in streptozotocin-induced (STZ) diabetic rats. Male Wistar strain (WNIN) rats (2months old; $213 \pm 14 \mathrm{gBW})$ were randomly assigned into 4 groups: control group (C), diabetic group (STZ-diabetic rats, D), STZ DM+ Soluble curcumin (SC, CurcuWIN ${ }^{\mathrm{TM}}$ ) and STZ DM + regular curcumin (RC). Daily food intake, weekly body weights, fasting glucose levels and $\mathrm{HbA1C}$ were assessed. Electroretinogram, immunohistochemistry and Immunofluorescence were performed in all treatment groups. At the end of 12 weeks, rats were euthanized and retinas harvested for histological and molecular analysis. In streptozotocin (STZ) diabetic rats (D) amplitude of oscillatory potentials (Ops) were reduced $(334.2 \mu \mathrm{V})$ compared to normal control (C) animals. Curcumin resulted in significant changes in OP amplitudes suggested by sum of OPs, RC (445.7) and SC (455.3). Treatment with curcumin prevented gross morphological changes to a significant extent in diabetic retina. Treatment with RC and SC prevented loss of Rhodopsin (Rho) protein expression and nerve growth factor (NGF) protein expression in diabetic retina indicated by intense fluorescence. Treatment with RC and SC showed decreased protein expression in diabetic retina indicated by lower Hypoxia-inducible factor 1-alpha (HIF-1- $\alpha$ ) positive fluorescence indicating the prevention of hypoxia in the retinas. Treatment with RC did not affect the vascular endothelial growth factor (VEGF) over expression in diabetic retina but the SC reduced the VEGF expression. In STZDM the mRNA levels of Glial fibrillary acidic protein (GFAP), are up regulated drastically, while treatment with SC significantly prevented its increase but there is no significant effect of RC treatment. Therefore, soluble curcumin may have therapeutic potential in the treatment of diabetic retinopathy (DR) and appears to be more effective than regular curcumin.
\end{abstract}

Keywords: diabetes mellitus, diabetic retinopathy, curcumin, electroretinogram
Volume 3 Issue I - 2015

\author{
Jayant Deshpande,' Shankarnarayanan \\ Jeykodi,' Bhanuprakash Reddy G, ${ }^{2}$ Sreenivasa \\ Reddy, ${ }^{2}$ Vijaya Juturu ${ }^{3}$ \\ 'OmniActive Health Technologies Ltd, India \\ ${ }^{2}$ National Institute of Nutrition, India \\ ${ }^{3}$ OmniActive Health Technologies Inc, USA
}

Correspondence: Vijaya Juturu, Manager, Scientific \& Clinical Affairs, OmniActive Health Technologies, Inc., 67 East Park Place, Morristown, NJ 07960 USA, Tel I-866-588-3629,

Email v.juturu@omniactives.com

Received: September 25, 2015 | Published: October 26, 2015
Abbreviations: STZ, streptozotocin; RC, regular curcumin; D, diabetic rats; Ops, oscillatory potentials; Rho, rhodopsin; NGF, nerve growth factor; HIF-1- $\alpha$, hypoxia-inducible factor 1-Alpha; vegf, vascular endothelial growth factor; GFAP, glial fibrillary acidic protein; T1D, type 1 diabetes; T2D, type 2 diabetes; CVD, cardiovascular disease; IR, insulin resistance; Nrf2, nuclear factor (erythroid-derived 2)-like; SC, soluble curcumin; RC, regular curcumin; ERG, electeroretinogram; PDGF, platelet-derived growth factor; HbAlc, glycated haemoglobin; INL, inner nuclear layer; OPL, outer plexiform layer; ONL, outer nuclear layer; ROS, reactive oxygen species; DRVS, the diabetic retinopathy vitrectomy study

\section{Introduction}

Diabetes mellitus (DM) is a chronic debilitating condition whose worldwide prevalence is rapidly increasing as a consequence of increases in obesity, changing patterns of diet and physical activity and ageing populations. The World Health Organization estimated that 154 million people in the world had DM at the beginning of the 21st century. ${ }^{1} \mathrm{DM}$ is a metabolic disorder characterized by hyperglycemia. Hyperglycemia is caused as a consequence of insulin deficiency in type 1 diabetes (T1D), and is a feature of late type 2 diabetes (T2D) along with insulin resistance. Diabetic retinopathy (DR) involves damage to the retina caused by the complications of diabetes, which can eventually lead to blindness.
DR occurs in both type 1 and type 2 diabetes mellitus and it has been shown that nearly all type 1 and $75 \%$ of type 2 diabetes will develop DR after a $15 \mathrm{yr}$ duration of as shown in previous epidemiological studies. ${ }^{2,3}$ Diabetes mellitus (DM) is a progressive disorder and is associated with cardiovascular disease (CVD) and metabolic risk such as central obesity, hypertension, insulin resistance (IR), dyslipidemia, micro albuminuria, and hyper coagulability. These risk factors increase diabetes related pathologies such as CVD, hypertension, peripheral artery disease, stroke, congestive heart failure, chronic kidney disease, and overall mortality. Impacting 25.8 million children and adults in the United States, diabetes is also associated with endothelial dysfunction, inflammation, abnormal thrombolysis and increased oxidative stress, which accentuate the progression of CVD. ${ }^{4}$

Retinal microvascular changes such as retinal arteriolar narrowing, venular dilation and isolated retinopathy are signs sometimes found in patients considered to be nondiabetic. Retinal microvascular signs have been shown to be associated with long-term risks of type 2 diabetes and hypertension, components of the metabolic syndrome (e.g. obesity, dyslipidemia) and a range of macro- and microvascular conditions (e.g. stroke, cardiovascular mortality). There is evidence that endothelial dysfunction and inflammation might be possible mechanisms involved in the development of various retinal microvascular changes in patients with diabetes, hypertension and other metabolic disorders. There are many changes to the eye that 
can occur with diabetes, especially if diabetes has been present for a long time. These can include cornea problems, glaucoma and/or cataracts. However, the most serious complication of diabetes to the eye is diabetic retinopathy. Most people living with diabetes more than 20years will develop diabetic retinopathy. ${ }^{5}$ Control of visionthreatening signs of DR such as retinal inflammation, progressive diabetic retinopathy, and light-induced retinal degeneration, diabetic macular edema and proliferative diabetic retinopathy are crucial for optimal clinical management of diabetes. The early identification of retinal microvascular changes are of prime importance in diagnosing the onset of diabetic retinopathy.

Curcumin has been identified as a natural compound having a plethora of biologic and pharmacologic properties owing to its antioxidant and anti-inflammatory activities. ${ }^{6-8}$ Curcumin has beneficial effects in in vitro and in vivo models on several ocular diseases, such as chronic anterior uveitis, diabetic retinopathy, glaucoma, age-related macular degeneration, and dry eye syndrome. ${ }^{9}$ Curcumin can activate the nuclear factor (erythroid-derived 2)-like 2 (Nrf2), which has been recognized as a master regulator of the antioxidant response. ${ }^{9}$ Its biological actions depend on its bioavailability and solubility. Most of the curcumin formulations are poorly absorbed, unstable at physiological $\mathrm{pH}$, rapidly metabolized, and systemically eliminated. Soluble curcumin ( $20 \%$ total curcuminoids) which was made water soluble by dispersing curcumin on a water-soluble carrier such as polyvinyl pyrrolidone and adding antioxidants to prevent degradation of curcumin during processing and storage. The STZ rat model of DM has been widely investigated since 1963 and is one of the most commonly used models of human disease. It is known to mimic many of the acute and some of the chronic complications observed in human diabetes. This model has the advantage of being highly reproducible and the time lines for various complications to develop are well recognized and reproducible. Given the established similarities of some of the structural, functional and biochemical abnormalities to human disease, it is considered an appropriate model to assess mechanisms of diabetes and evaluate potential therapies. Therefore, the present study aims to investigate the effect of soluble curcumin on diabetes-induced changes in glucose metabolism and molecular biomarkers including rhodopsin, nerve growth factor, Hypoxiainducible factor 1-alpha, vascular endothelial growth factor (VEGF), and Glial fibrillary acidic protein (GFAP) protein expressions using STZ-induced diabetic rat model.

\section{Methodology}

\section{A. Experimental design}

Male Wistar strain (WNIN) rats (2 months old; Average BW of $213 \pm 14 \mathrm{~g}$ ) were obtained from the National Center for Laboratory Animal Sciences, National Institute of Nutrition, Hyderabad, India (NCLAS, NIN). Animals were maintained at NCLAS, NIN and kept for acclimatization in an experimental room for two weeks. A novel water-soluble curcumin (SC) formulation ( $20 \%$ total curcuminoids, OmniActive Health Technologies, Thane, India) which was made containing turmeric extract $20-28 \%$, a hydrophilic carrier $63-75 \%$ (polyvinyl pyrrolidone), cellulosic derivatives $10-40 \%$ and natural antioxidants $1-3 \%$. Regular curcumin (RC) contains 95\% total curcuminoids. Table 1 provides dose information for each treatment. Diabetes was induced in overnight fasted animals by a single intraperitoneal injection of STZ $(30 \mathrm{mg} / \mathrm{kg})$ in $0.1 \mathrm{M}$ citrate buffer, $\mathrm{pH}$ 4.5. Another set of rats, which received only the vehicle, served as the control (Group I; $n=12$ ). Fasting blood glucose levels were measured
$72 \mathrm{~h}$ after STZ injection. Animals having blood glucose levels $>150$ $\mathrm{mg} / \mathrm{dL}$ were considered diabetic and all animals were divided into four groups (Group I-IV, Table 1). All the animals were housed in individual cages and maintained on their respective diets for 12 weeks. Drinking water was provided ad libitum throughout the study period. Daily food intake and weekly body weights, fasting glucose levels were recorded. Before sacrifice electroretinograms were performed and $\mathrm{HbA} 1 \mathrm{C}$ levels were estimated. At the end of 12 weeks rats were euthanized and retinas harvested for histological and molecular study.

\section{B. Electeroretinogram (ERG) analysis}

Diabetic retinopathy was characterized by disturbances in retinal function. The function of the retina was assessed by electroretinogram. Diabetes results in ischemia and apoptosis in different retinal cell layers which result in changes in the functions of the retina. Oscillatory potentials (OPs) were more affected in diabetes than a- or b- waves. OPs represent the functional aspects of inner retinal layers, ganglion cell layer and the inner plexiform layer.

Table I Experimental design of treatment groups

\begin{tabular}{|c|c|c|}
\hline & Group & Diet \\
\hline I & Control, $\mathrm{N}=6$ & AIN 93 \\
\hline II & Diabetic, $N=9$ & AIN $93+$ STZ \\
\hline III & Diabetic + SC, $N=8$ & AIN 93 with soluble curcumin $0.01 \%$ \\
\hline IV & Diabetic $+R C, N=6$ & AIN 93 with regular curcumin $0.01 \%$ \\
\hline
\end{tabular}

AIN93, standard diet; STZ, streptozotocin; SC, soluble curcumin (CurcuWINTM); RC, regular curcumin

Animals were dark-adapted overnight and prepared for the ERG procedure under dim red illumination. The pupils of the rats were dilated with atropine eye drops. The ground electrode was a subcutaneous needle in the tail, and the reference electrode was an ear clip electrode. The active contact lens electrodes were placed on the cornea. The recordings were performed with a UTAS Visual Diagnostic System. The responses were differentially amplified with a gain of 1,000 using alternating current-coupled UBA-4204 Amplifier. A flash stimuli of -2 to $8 \mathrm{~dB}$ were delivered via a with BigShot ${ }^{\mathrm{TM}}$ Ganzfeld System (LKC Technologies; Gaithersburg, MD, USA). The oscillatory potentials were extracted from the wave form and the sum of all OPs was calculated.

i. Histopathology: The eyeballs from selected animals were collected in a fixative of $4 \%$ paraformaldehyde solution in separately labeled vials. The tissues were given some nicks so as to facilitate penetration of the fixative into deep tissue. They were kept at room temperature for $24-48 \mathrm{hrs}$ followed with replacing the fixative with $20 \mathrm{mM}$ sodium phosphate buffer. The buffer was scheduled to be replaced with fresh buffer once weekly until histopathological processing. Tissues were embedded in paraffin and sections were taken in microtome. Coated slides were used for immunohistochemistry and immunofluorescence, whereas uncoated slides were used for $\mathrm{H} \& \mathrm{E}$ staining.

ii. Quantitative real time PCR: We isolated RNA from the rats and quantified mRNA of various genes including nerve growth factor (NGF), glial acidic fibrillary protein (GFAP), vascular endothelial growth factor (VEGF), rhodopsin, hypoxia-inducible factor (HIF) and platelet-derived growth factor (PDGF) in the retina of experimental rats by means of quantitative real time PCR. ${ }^{9-11}$ 
iii. Immunohistochemistry: Retinas were fixed in 4\% paraformaldehyde in sodium phosphate buffer ( $\mathrm{pH}$ 7.2), followed by embedding and sectioning for paraffin using standard protocols. Three retinas were used for each group. Immunolocalization of GFAP, VEGF and rhodopsin were carried out on $4 \mu \mathrm{M}$ thick paraffin sections of rat retinas. The protein was localized by staining in the retina sections by addition of $\mathrm{DAB}$ solution containing $\mathrm{H}_{2} \mathrm{O}_{2}$ or fluorescent tagged antibodies. Slides were observed under a microscope (\#LMD 6000, Leica Microsystems, Germany). The complete retinal morphology including thickness of various layers was studied in detail in the retinal sections.

iv. Immunoblotting: In addition to immunohistochemistry, we quantified protein levels of VEGF, GFAP and rhodopsin in the retinas of experimental rats using immunoblotting. Housekeeping protein $\beta$-actin was used as a loading control. Quantification of band intensity was done using Image J software.

v. Statistical analysis: The data were expressed as mean \pm SEM. Results were analyzed for statistical significance by one way ANOVA followed by Dunnett's multiple comparison tests for comparing all the groups with the control group. Between group significance was checked by two tailed unpaired t-test. All analyses were performed with significance set at $P$ less than 0.05 .

\section{Results}

\section{A. Body weights and average daily food intake}

The diabetic control rats were found to have significantly reduced body weight $(\mathrm{p}<0.001)$ compared to age matched non-diabetic control rats. The diabetic control rats consumed significantly $(\mathrm{p}<0.001)$ more food than the non-diabetic control rats (Figure 1A\&B). Despite the increased food intake, the body weight of diabetic (D) animals were significantly decreased when compared with age matched non diabetic controls $(\mathrm{C})(\mathrm{P}<0.001)$.

\section{B. Fasting blood glucose}

The fasting plasma glucose concentrations of the diabetic control rats were significantly higher than those of the non-diabetic control rats throughout the experiment. There was a marginal decrease in fasting plasma glucose levels observed in groups treated with SC but there was no significant effect of treatment on fasting plasma glucose in diabetic rats (Figure 1C).

\section{Glycated haemoglobin (HbA1c)}

Glycated haemoglobin (HbAlc) was a better indicator than fasting blood glucose levels of the glycemic status in diabetics. A significant increase in HbAlc levels were observed in diabetic animals $(>6.5$ $\%)$. Treatment with SC was found to decrease the HbAlc percentage moderately but it was not statistically significant (Figure 1D). These results were found to be in correlation with fasting blood glucose pattern between groups. No significant changes were observed with RC.

\section{Diabetic retinopathy}

Electroretinograph: In diabetic rats (D) amplitude of OPs were reduced $(334.2 \mu \mathrm{V})$ compared to normal control $(\mathrm{C})$ animals. Ingestion of curcumin resulted in lowering the reduction in OP amplitudes suggested by sum of Ops compared with RC (445.7) and SC (455.3). $\mathrm{SC}$ further improved the implicit times (Figure 2).

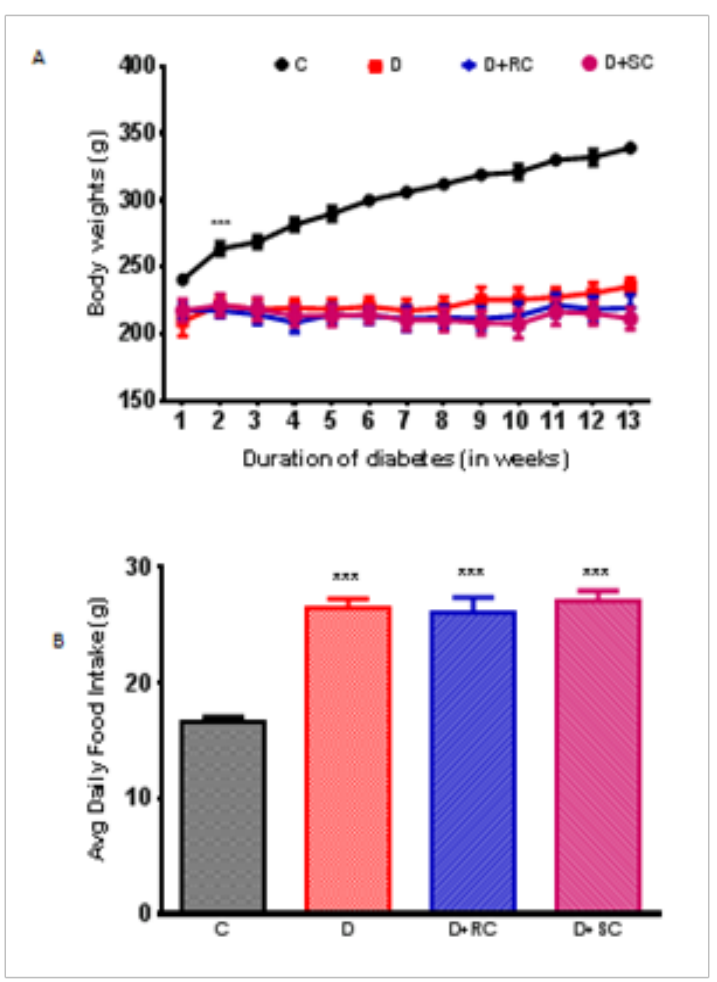

Figure I A\&B Body weight and feed intake of rats in different treatments.
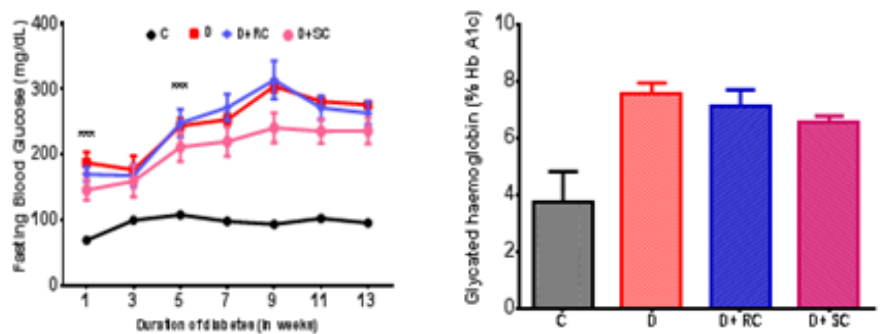

Figure IC\&D Blood glucose and glycosylated hemoglobin in different treatments.

H \& E Staining: In control rats (C), all retinal layers were intact with maximum thickness of retina. Also noted was a dense inner nuclear layer (INL) and distinguishable separation of outer plexiform layer (OPL) between INL and outer nuclear layer (ONL). In contrast, retinas of diabetic rats (D) showed significantly reduced total retinal thickness and were marked by less dense ONL and almost merged INL and ONLs. Treatment with either curcumin prevented gross morphological changes to a significant extent in diabetic retina. However, soluble formulations of these two active principles were shown to be more effective than the regular active principles, indicated by increased density of ONLs.

\section{E. Immunofluorescence}

Rhodopsin (Rho): Rhodopsin is a biological pigment in photoreceptor cells of the retina responsible for the first events in the perception of light. Immunofluorescence imaging of Rho protein showed its decreased expression in diabetic rat retina in 
comparison to normal control rat retina. Treatment with $\mathrm{RC}$ and SC prevented loss of Rho protein expression in diabetic retina indicated by intensed Rho positive fluorescence. Furthermore, both RC and SC resulted in prevention of loss of Rho protein expression in rat retina in diabetic animals respectively (Figure 3A).

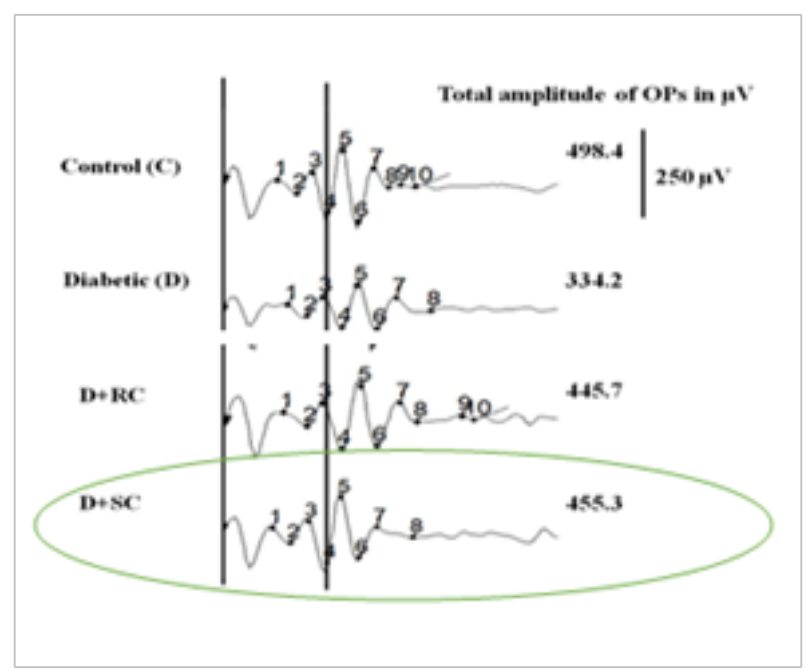

Figure 2 Soluble curcumin shows an increase in total amplitude of Ops compared with diabetic rats. C, Non diabetic control; D, Diabetic control; $D+R C$, diabetes+regular curcumin; $d+s c$, diabetes+soluble curcumin.

f. Nerve growth factor (NGF): Immunofluorescence imaging of NGF protein showed its decreased expression in diabetic rat's retina in comparison to normal control rat's retina (Figure 3). Treatment with RC and SC prevented loss of NGF protein expression in diabetic retina indicated by intense NGF positive fluorescence. The mRNA levels of NGF in the retinal of various experimental groups are shown in the above graph (Figure 3B). The results were consistent with that of protein level shown by immuno fluorescence.

g. Hypoxia-inducible factor 1-alpha (HIF-1-alpha): HIF $1 \alpha$ is expressed at low oxygen concentrations and plays an essential role in cellular and systemic responses to hypoxia. Immuno fluorescence imaging of HIF1 $\alpha$ protein increased expression was observed in diabetic rat's retina in comparison to normal control rat retina in various layers (Figure 3C). Treatment with RC and SC decreased protein expression in diabetic retina indicated by lower HIF $1 \alpha$ positive fluorescence indicating the reduction of hypoxia.

h. Western blotting for VEGF:Vascular endothelial growth factor (VEGF) is a signal protein produced by cells that stimulates vasculogenesis and angiogenesis. When VEGF is over expressed, it can contribute to disease condition. Western blotting for VEGF indicated up regulation of VEGF expression in diabetic retina (Figure $4 \mathrm{~A} \& \mathrm{~B})$. Treatment with RC did not affect VEGF over expression in diabetic retina but treatment with SC reduced VEGF over expression.

i. Glial fibrillary acidic protein (GFAP): GFAP is involved in many important central nervous system processes, including cell communication, cell strength and shape and the functioning of the blood brain barrier. In diabetic rats the mRNA levels of GFAP levels were up regulated drastically. While treatment with SC significantly prevented its increase, there was no significant effect of $\mathrm{RC}$ treatment.
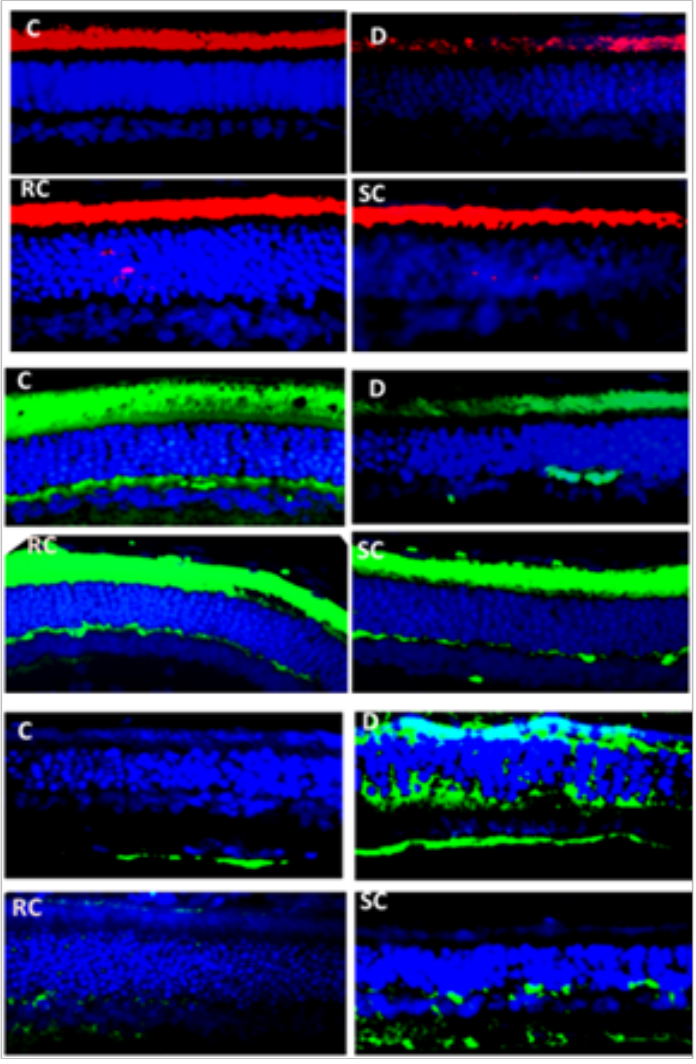

Figure 3 Immunofluorescence changes in different gene proteins in retina. (A) Rhodopsin (Rho). (B) Nerve growth factor (NGF). (C) Hypoxia-inducible factor I-alpha (HIF-I-alpha).

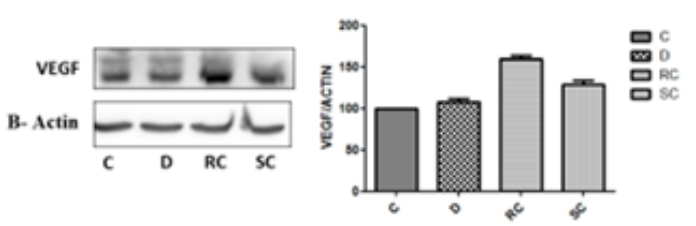

Figure 4A Vascular endothelial growth factor (VEGF).
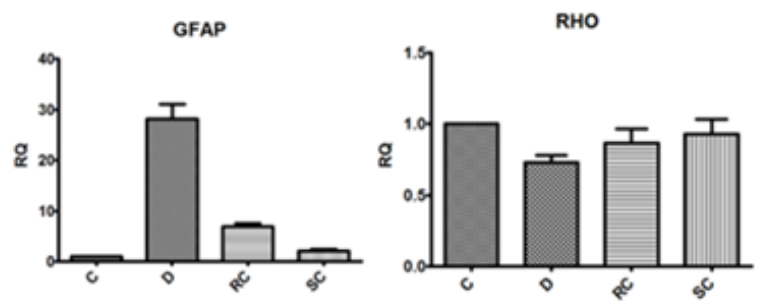

Figure 4B Soluble curcumin decreased Glial fibrillary acidic protein (GFAP) and increased Rhodopsin. 


\section{Discussion}

Oxidative stress is believed to play a significant role in the development of diabetic retinopathy. There are two eye diseases that result from diabetic micro vascular complications: diabetic retinopathy and macular edema. The pathogenesis of macular edema appears to be multi factorial. It corresponds to a swelling in the macula which is a yellow area in the central retina responsible for detailed central vision. The dilated vessels are generally leaky and fluid builds up in the macula. This process in turn causes the macula to swell and cease to function. It is the most common cause of visual impairment in patients with non-proliferative diabetic retinopathy.

The role of vascular endothelial growth factor appears to be central in the pathogenesis of proliferative diabetic retinopathy. Advanced glycation end products are important in the development of vitreous abnormalities in proliferative diabetic retinopathy. In the present study, we have shown that curcumin supplementation in the diabetes group (D) reduced oxidative stress and improved antioxidant capacity. It is well established that hyperglycemia can produce ROS by a series of cellular events and further leads to diabetic complications due to oxidative stress. ${ }^{12,13}$ The Early Treatment Diabetic Retinopathy Study showed that patients with type II diabetes mellitus who were older than 40 with severe non proliferative diabetic retinopathy (defined as hemorrhages in four quadrants, venous beading in two quadrants, or intra-retinal microvascular abnormalities in one quadrant) also benefited from early retinal photocoagulation. ${ }^{14}$ The Diabetic Retinopathy Vitrectomy Study (DRVS) research group showed that early surgery in patients with proliferative DM is beneficial as they tend to develop aggressive fibro vascular proliferation. It was observed that $25 \%$ of patients undergoing early vitrectomy recovered visual acuity (approximately 20/40 or better).

Curcumin has been demonstrated to activate Nrf2 in obesity, metabolic syndrome, nephropathy, retinopathy and neuropathy, where its activation prevents the development of diabetes and its complications. The Diabetic Retinopathy Vitrectomy Study showed that early vitrectomy (within 6 months of onset of vitreous hemorrhage) was associated with better results in type I diabetes mellitus patients. ${ }^{15}$ Curcumin was shown to induce apoptosis, decrease VEGF, inhibit diabetes-induced elevation of serum VEGF levels and rescue photoreceptor degeneration in transgenic rats with $\mathrm{P} 23 \mathrm{H}$ rhodopsin mutation. ${ }^{16,17}$ Curcumin has been reported to inhibit the generation of hydroxyl radicals (.OH) to an extent of $76 \%$ and $70 \%$ and prevent the oxidation of $\mathrm{Fe}^{2+}$ in Fentons reaction which generates .OH radicals. ${ }^{18} \mathrm{NGF}$ is important regulator of retinal development and also plays a key role in regulating survival, growth, and functional maintenance of retinal ganglion cells, photoreceptors and other retinal neurons. GFAP is an intermediate filament present in retinal glial cells (ganglion cells) and retinal astrocytes that maintains structure and function of the cell's cytoskeleton. VEGF regulates angiogenesis in the retina. Rhodopsin is responsible for both the formation of the photoreceptor cells and the first events in the perception of light. HIF1alpha is the master regulator of cellular adaptation to low oxygen tension. PDGF plays an important role in development of the central nervous system, including the retina. Excessive PDGF signaling is associated with proliferative retinal disorders.

The phenolic $(\mathrm{OH})$ structure of curcumin is believed to be essential for its anti-oxidant activity. ${ }^{19}$ Our findings are consistent with others showing that treatment with curcumin in diabetic rat's leads to lower plasma glucose levels. ${ }^{20,21}$ These microarray results provide clues to understanding the molecular pathways underlying photoreceptor degeneration and indicate directions for future studies. Curcumin suppressed oxidative stress and showed a beneficial effect. Curcumin may have a protective role against oxidative stress and may reverse diabetes-induced oxidative retinal injury.

Diabetes-associated metabolic abnormalities directly impair neural retinal function before the development of vasculopathy, thereby resulting in visual deficits. The Diabetic Retinopathy Study demonstrated the ability of retinal photocoagulation to reduce the rate of severe visual loss by $50 \%$ for eyes with high-risk characteristics, defined as neo vascularization originating from the optic disk $>1 / 3$ disk diameter, any neovascularization originating from the optic disk with hemorrhage, and neo vascularization originating from the retina with vitreous hemorrhage. ${ }^{22,23}$ Antonetti et al., \& Klein et al., ${ }^{24,25}$ reported that hypoxia inducible factor-1 (HIF-1) and vascular endothelial growth factor (VEGF) play an important role in the pathogenesis of DR and neovascularization..$^{26,27}$

Curcumin bioavailability and absorption are challenging research issues and are important to achieving the potential therapeutic benefits of curcumin. Recently, Jaeger et al. ${ }^{28}$ reviewed and reported bioavailability and absorption of different forms of curcumin in animal studies. For example, curcumin with an extract obtained from the black pepper increased absorption by 1.5 -fold, a complex of curcumin with phospholipids increased absorption by 3.4-fold, and a formulation of curcumin with a micellar surfactant (polysorbate) in mice increased absorption by 9.0 -fold. Also, a micro emulsion system of curcumin [Capryol 90 (oil), Cremophor RH40 (surfactant), and Transcutol P aqueous solution (co-surfactant)] increased absorption by 22.6-fold and Polylactic-co-glycolic acid (PLGA) and PLGA-polyethylene glycol (PEG) (PLGA-PEG) blend nanoparticles increased absorption by $15.6-$ and 55.4 -fold, respectively.

Most curcumin studies fail to show efficacy, ${ }^{29,30}$ because of poor bioavailability resulting from limited solubility of curcumin in an aqueous medium. Ringman et al., ${ }^{30}$ reported a study in patients with mild-to-moderate Alzheimer's disease $(\mathrm{AD})$ that were randomized to receive placebo, 2 grams/day, or 4grams/day of oral curcumin for 24weeks. Curcumin treatment was associated with lowered hematocrit and increased glucose levels. There were no differences between treatment groups in clinical or biomarker efficacy measures. In a recent study, ${ }^{28}$ soluble curcumin (SC) significantly increased curcuminoid appearance in the blood compared with other formulations. Therefore, water-soluble curcumin preparations are likely more effective and may increase potential therapeutic benefits. In addition, tocopherol and ascorbyl palmitate were used to prevent degradation of curcumin during processing and storage. Based on the AMES test, including 14 day, 28 day and 90 day toxicity studies in animals, SC is safe. No adverse effects were observed in animal and human studies (unpublished). In the United States, turmeric is generally recognized as safe (GRAS) by the FDA as a food additive, ${ }^{31}$ and The Joint FAO/WHO Expert Committee on Food Additives stipulated an acceptable daily intake (ADI) of $50 \mathrm{mg} / \mathrm{kg}$ body weight for polyvinylpyrrolidone. ${ }^{32}$ Serious adverse effects have not been reported in humans taking curcumin supplementation up to $12 \mathrm{~g}$ /day ${ }^{33,34}$ Further human clinical trials designed to study the effect of curcumin on specific health markers are required in healthy population to validate the health benefits. Finally, in designing human clinical trials, it is important to differentiate between those targeted for prevention and others targeted for treatment. Treatment trials include duration and diagnosis of condition and medication use and cannot be 
extrapolated to healthy human populations. More prevention targeted trials are required to explore the potential health benefits in a healthy human population. Curcumin inhibits diabetic retinal oxidative stress, reversing diabetes-induced up-regulation of retinal Glial fibrillary acidic protein (GFAP). Curcumin is potentially a safe and inexpensive substitute (or adjunctive) treatment in the management of diabetic eye disease.

\section{Conclusion}

These results suggest soluble curcumin has a therapeutic potential in the treatment of diabetic retinopathy (DR) and is more effective than regular curcumin. Curcumin supplementation may benefit diabetic patients by improving microvascular complications and preventing retinal disease in diabetes conditions. Further, soluble curcumin may be explored to prevent or delay the development of ocular diseases (including diabetic retinopathy) and other diabetes related pathologies.

\section{Acknowledgments}

None.

\section{Conflicts of interest}

The author declares that there are no conflicts of interest.

\section{Funding}

None.

\section{References}

1. King H, Aubert RE, Herman WH. Global burden of diabetes, 1995 2025: prevalence, numerical estimates, and projections. Diabetes Care. 1998;21(9):1414-1431.

2. Klein R, Klein BE, Moss SE, et al. The Wisconsin Epidemiologic Study of Diabetic Retinopathy. II. Prevalence and risk of diabetic retinopathy when age at diagnosis is less than 30 years. Arch Ophthalmol. 1984;102(4):520-526.

3. Klein R, Klein BEK, Moss SE, et al. The Wisconsin epidemiologic study of diabetic retinopathy III. Prevalence and risk of diabetic retinopathy when age at diagnosis is 30 or more years. Arch Ophthalmol. 1984;102(4):527-532.

4. Johnson AR, Milner JJ, Makowski L. The inflammation highway: metabolism accelerates inflammatory traffic in obesity. Immunol Rev. 2012;249(1):218-238.

5. International Diabetes Federation, IDF World Atlas Sixth Edition, 'The Global Burden'. 2014.

6. Kaufman SC, Ferris FL, Swartz M. The Diabetic Retinopathy Study Research Group: Intraocular pressure following panretinal photocoagulation for diabetic retinopathy. Diabetic Retinopathy Study (DRS) Report Number 11. Arch Ophthalmol. 1987;97:807-809.

7. Flynn HW, Chew EY, Simmons BD, et al. Pars plana vitrectomy in the Early Treatment Diabetic Retinopathy Study. ETDRS report number 17. The Early Treatment Diabetic Retinopathy Study Research Group. Ophthalmology. 1992;99(9):1351-1357.

8. Early vitrectomy for severe vitreous hemorrhage in diabetic retinopathy Two-year results of a randomized trial. Diabetic Retinopathy Vitrectomy Study report 2. The Diabetic Retinopathy Vitrectomy Study Research Group. Arch Ophthalmol. 1985;103(11):1644-1652.
9. Jeenger MK, Shrivastava S, Yerra VG, et al. Curcumin: A pleiotropic phytonutrient in diabetic complications. Nutrition. 2015;31(2):276282.

10. Tiruvalluru M, Ananthathmakula P, Ayyalasomayajula V, et al. Vitamin A supplementation ameliorates obesity associated retinal degeneration in WNIN/Ob rats. Nutrition. 2013;29(1):298-304.

11. Vasireddy V, Chavali VR, Joseph VT, et al. Rescue of photoreceptor degeneration by curcumin in transgenic rats with $\mathrm{P} 23 \mathrm{H}$ rhodopsin mutation. PLoS One. 2011;6(6):e21193.

12. Reddy GB, Vasireddy V, Mandal MN, et al. A novel rat model with obesity-associated retinal degeneration. Invest Ophthalmol Vis Sci. 2009;50(7):3456-3463.

13. Klein R, Klein BE, Moss SE, et al. The Wisconsin Epidemiologic Study of Diabetic Retinopathy. II. Prevalence and risk of diabetic retinopathy when age at diagnosis is less than 30 years. Arch Ophthalmol. 1984;102(4):520-526.

14. Kaufman SC, Ferris FL, Swartz M. The Diabetic Retinopathy Study Research Group: Intraocular pressure following panretinal photocoagulation for diabetic retinopathy. Diabetic Retinopathy Study (DRS) Report Number 11. Arch Ophthalmol. 1987;97:807-809.

15. Photocoagulation for diabetic macular edema. Early Treatment Diabetic Retinopathy Study report number 1. Early Treatment Diabetic Retinopathy Study research group. Arch Ophthalmol. 1985;103(12):1796-806.

16. Early vitrectomy for severe vitreous hemorrhage in diabetic retinopathy. Four-year results of a randomized trial: Diabetic Retinopathy Vitrectomy Study Report 5. Arch Ophthalmol. 1990;108(7):958-964.

17. Premanand C, Rema M, Sameer MZ, et al. Effect of curcumin on proliferation of human retinal endothelial cells under in vitro conditions. Invest Ophthalmol Vis Sci. 2006;47(5):2179-2184.

18. Kowluru RA, Kanwar M. Effects of curcumin on retinal oxidative stress and inflammation in diabetes. Nutr Metab (Lond). 2007;4:8.

19. Reddy AC, Lokesh BR. Studies on the inhibitory effects of curcumin and eugenol on the formation of reactive oxygen species and the oxidation of ferrous iron. Mol Cell Biochem. 1994;137(1):1-8.

20. Priyadarsini KI, Maity DK, Naik GH, et al. Role of phenolic O-H and methylene hydrogen on the free radical reactions and antioxidant activity of curcumin. Free Radic Biol Med. 2003;35(5):475-484.

21. Sharma S, Kulkarni SK, Chopra K. Curcumin, the active principle of turmeric (Curcuma longa), ameliorates diabetic nephropathy in rats. Clin Exp Pharmacol Physiol. 2006;33(10):940-945.

22. Patumraj S, Wongeakin N, Sridulyakul P, et al. Combined effects of curcumin and vitamin $\mathrm{C}$ to protect endothelial dysfunction in the iris tissue of STZ-induced diabetic rats. Clin Hemorheol Microcirc. 2006;35(4):481-489.

23. Gündüz K, Bakri SJ. Management of proliferative diabetic retinopathy. Compr Ophthalmol Update. 2007;8(5):245-256.

24. Antonetti DA, Barber AJ, Hollinger LA, et al. Vascular endothelial growth factor induces rapid phosphorylation of tight junction proteins occludin and zonula occluden 1. A potential mechanism for vascular permeability in diabetic retinopathy and tumors. $J$ Biol Chem. 1999;274(33):23463-23467.

25. Klein R, Knudtson MD, Lee KE, et al. The Wisconsin Epidemiologic Study of Diabetic Retinopathy: XXII the twenty-five-year progression of retinopathy in persons with type 1 diabetes. Ophthalmology. 2008;115(11):1859-1868. 
26. Klein R, Klein BE. Is the prevalence of visual impairment rising or falling in the people with diabetes mellitus? It depends on who you study. JAMA Ophthalmol. 2013;131(7):948-950.

27. Stewart MW. The expanding role of vascular endothelial growth factor inhibitors in ophthalmology. Mayo Clin Proc. 2012;87(1):77-88.

28. Jäger R, Lowery RP, Calvanese AV, et al. Comparative absorption of curcumin formulations. Nutr J. 2014;13:11.

29. Devassy JG, Nwachukwu ID, Jones PJ. Curcumin and cancer: barriers to obtaining a health claim. Nutr Rev. 2015;73(3):155-165.

30. Ringman JM, Frautschy SA, Teng E, et al. Oral curcumin for Alzheimer's disease: tolerability and efficacy in a 24-week randomized, double blind, placebo-controlled study. Alzheimers Res Ther. 2012;4(5):43.
31. US Food and Drug Administration. Food Additive Status List. 2005.

32. JECFA. 30th report of the Joint FAO/WHO Expert Committee on Food Additives, WHO Technical report series. Geneva. 1987;751.

33. Lao CD, Ruffin MT, Normolle D, et al. Dose escalation of a curcuminoid formulation. BMC Complement Altern Med. 2006;6:10.

34. Cheng AL, Hsu CH, Lin JK, et al. Phase I clinical trial of curcumin, a chemopreventive agent in patients with high-risk or pre-malignant lesions. Anticancer Res. 2001;21(4B):2895-2900. 